\title{
BMJ Open Using cluster analysis to describe phenotypical heterogeneity in extremely preterm infants: a retrospective whole- population study
}

\author{
Theodore Dassios (D) , ${ }^{1,2}$ Emma E Williams, ${ }^{1}$ Christopher Harris, ${ }^{1,2}$ \\ Anne Greenough (D) 1,3
}

To cite: Dassios T, Williams EE, Harris C, et al. Using cluster analysis to describe phenotypical heterogeneity in extremely preterm infants: a retrospective wholepopulation study. BMJ Open 2022;12:e56567. doi:10.1136/ bmjopen-2021-056567

- Prepublication history for this paper is available online. To view these files, please visit the journal online (http://dx.doi org/10.1136/bmjopen-2021 056567).

Received 18 August 2021 Accepted 12 February 2022

D) Check for updates

(C) Author(s) (or their employer(s)) 2022. Re-use permitted under CC BY-NC. No commercial re-use. See rights and permissions. Published by BMJ.

${ }^{1}$ Department of Women and Children's Health, School of Life Sciences, Faculty of Life Science and Medicine, King's College London, London, UK

${ }^{2}$ Neonatal Intensive Care Centre, King's College Hospital NHS Foundation Trust, London, UK ${ }^{3}$ Asthma UK Centre for Allergic Mechanisms in Asthma, King's College London, London, UK

Correspondence to

Dr Theodore Dassios;

theodore.dassios@kcl.ac.uk

\section{ABSTRACT}

Objective To use cluster analysis to identify discrete phenotypic groups of extremely preterm infants.

Design Secondary analysis of a retrospective whole population study.

Setting All neonatal units in England between 2014 and 2019.

Participants Infants live-born at less than 28 weeks of gestation and admitted to a neonatal unit.

Interventions $\mathrm{K}$-means cluster analysis was performed with the gestational age, Apgar score at $5 \mathrm{~min}$ and duration of mechanical ventilation as input variables.

\section{Primary and secondary outcome}

measures Bronchopulmonary dysplasia, discharge on home oxygen, intraventricular haemorrhage, death before discharge from neonatal care.

Results Ten thousand one hundred and ninety-seven infants $(53 \%$ male) were classified into four clusters:

Cluster 1 contained infants with intermediate gestation and duration of ventilation and had an intermediate mortality and incidence of bronchopulmonary dysplasia. Cluster 2 contained infants with the highest gestation, a shorter duration of ventilation and the lowest mortality. Cluster 3 contained infants with the lowest Apgar score and highest mortality and incidence of intraventricular haemorrhage. Cluster 4 contained infants with the lowest gestation, longest duration of ventilation and highest incidence of bronchopulmonary dysplasia.

Conclusion Clinical parameters can classify extremely preterm infants into discrete phenotypic groups with differing subsequent neonatal outcomes.

\section{INTRODUCTION}

Preterm birth is a major cause of childhood morbidity and mortality with a global incidence of approximately $10.6 \% .^{1}$ Infants born at less than 28 completed weeks of gestation (extremely preterm) suffer significant multisystem morbidity lasting into adolescence and adulthood, ${ }^{2}{ }^{3}$ with important financial implications for health systems ${ }^{4}$ and an increased physical, emotional and financial burden for the families. ${ }^{5}$ The survival of these infants has increased over the past decades ${ }^{6}$
Strengths and limitations of this study

- First study to use cluster analysis to classify extremely preterm infants in different phenotypical groups.

- We used readily available clinical parameters to classify extremely preterm infants into distinct phenotypic groups.

- The ensuing groups had differing neonatal outcomes such as survival to discharge from neonatal care and bronchopulmonary dysplasia.

- We used the whole population rather than a representative sample, making our results more generalisable.

- Our cluster analysis is not an early prediction study as we have included as input variable the duration of ventilation, which might be prolonged in the life of an extremely preterm infant.

and the associated implications for patients and families are only expected to increase in the future.

Although many extremely preterm infants suffer significant perinatal and long-term morbidity, ${ }^{7}$ there is substantial variation in the severity of the neonatal outcomes with a sizeable proportion escaping major morbidity. For example, we have recently reported the overall mortality and incidence of bronchopulmonary dysplasia (BPD) of extremely preterm infants in England to be $18.9 \%$ and $57.5 \%$, respectively, which means that $81.1 \%$ of those infants survived to discharge and $23.6 \%$ were free of BPD. ${ }^{8}$ Although the multifactorial origin of adverse neonatal outcomes has been well described, ${ }^{9}$ the heterogeneity in neonatal outcomes is poorly understood.

The use of unsupervised learning based on big-data analytics has been used in the clinical domain for the prediction of individual risk factors and for clinical decision support. ${ }^{10}$ Unsupervised learning could also be helpful 
to identify different phenotypic groups (clusters) corresponding to distinct clinical phenotypes of extreme preterm infants. Cluster analysis as a method of detecting subgroups within multidimensional data sets could also be helpful in identifying subgroups of patients that might benefit from targeted interventions. ${ }^{11} 12$ To our knowledge, such unsupervised learning approaches have not been previously employed to describe different phenotypical groups in extreme prematurity.

We aimed to derive and describe clusters of extremely preterm infants based on readily available clinical information and to explore whether these distinct phenotypes were associated with different neonatal outcomes.

\section{MATERIALS AND METHODS}

\section{Study design and participants}

All infants live-born before 28 completed weeks of gestation and admitted to a neonatal unit in England between 1 January 2014 and 1 January 2019 (study period) were included. This was an analysis of data previously acquired to investigate the relationship of growth impairment with the development of BPD in a retrospective, wholepopulation study. ${ }^{8}$ A predefined data set was acquired from the National Neonatal Research Database (NNRD), Imperial College London, UK. As the study used data held in an existing database, participation did not require approval from individual Trusts, but only from the NHS Trust holding the database (Chelsea and Westminster NHS Foundation Trust) which was obtained.

The following variables were collected: maternal age (years), administration of antenatal steroids (yes/no), gestational age at birth calculated from the last menstrual period or ultrasonographically (weeks), birth weight $(\mathrm{kg})$, Apgar score at $5 \mathrm{~min}$ of age, sex (male/female), duration of invasive ventilation (days), BPD development defined as any need for respiratory support at 36 weeks postmenstrual age (PMA), ${ }^{14}$ administration of postnatal corticosteroids (defined as parenteral administration of dexamethasone or hydrocortisone for more than 5 consecutive days-yes/no), surgical intervention for necrotising enterocolitis (yes/no) ${ }^{15}$ surgical ligation of patent ductus arteriosus (yes/no), ${ }^{16}$ intraventricular haemorrhage (IVH) grade $3-4$ (yes/no), ${ }^{17}$ periventricular leucomalacia (yes/no), ${ }^{18}$ death before discharge from neonatal care (yes/no), age at death (days), weight at 36 weeks PMA (kg), PMA at discharge (weeks), weight at discharge (kg), discharged on home oxygen (yes/no). We calculated the birth weight z-score $(\Delta \mathrm{Wz})$ using the UK-WHO preterm reference chart ${ }^{19}$ and the Microsoft Excel add-in LMS Growth (V.2.77; www.healthforchildren.co.uk). The birth weight z-score was not calculated for infants born $<23$ completed weeks of gestation, as there were no reference data.

\section{PPI statement}

This is a retrospective study, so patients and public could not be involved.

\section{Analysis}

Cluster analysis was performed with the smallest possible number of continuous variables necessary to adequately characterise neonatal outcomes, since inclusion of a large number of variables might degrade the final classification. ${ }^{11}{ }^{20} \mathrm{~K}$-means cluster analysis was performed with gestational age, Apgar score at 5 min and duration of mechanical ventilation as input variables. These parameters were selected, as they were independent, contributory to neonatal outcomes and pathophysiologically distinct between them. All results were standardised as z-scores prior to clustering. To select the number of clusters, clustering was performed for solutions comprising 2 to 10 clusters and the optimal solution was selected based on the minimum number of iterations that changed the cluster centres and the optimal $\mathrm{F}$ factor and significance of the corresponding Analysis of Variance (ANOVA) analysis. The $\mathrm{F}$ factor for each ANOVA cluster solution was calculated as the ratio of two variances, and a higher $\mathrm{F}$ factor corresponded to a stronger separation of the clusters. ${ }^{21}{ }^{22}$ Subjects with missing values in any of the three input variables were excluded from the analysis. Continuous variables were compared across clusters using Kruskal-Wallis with Mann-Whitney U test as a post hoc test for pairwise comparisons. Categorical variables were compared across clusters using $\chi^{2}$ test with Bonferroni adjustment as a post hoc test for pairwise comparisons. Cluster profiles were presented graphically using radial plots, where the length of each 'spoke' was proportional to the magnitude of the standardised variable. The clustering was also visualised using a discriminant-coordinates biplot, generated by canonical variate analysis, which projects multidimensional data into a lower dimensional space while preserving as much information as possible, to provide an easily interpretable two-dimensional representation of cluster separation and density.

Cluster and statistical analysis were performed using SPSS software, V.26.0 (IBM, Armonk, New York).

\section{RESULTS}

During the period of the study, 11806 infants were born alive below 28 completed weeks of gestation and admitted to a neonatal unit in England. One thousand five hundred and eighty-five infants were excluded for missing data on Apgar score in $5 \mathrm{~min}$. Twenty-one infants were removed for missing data on the duration of mechanical ventilation and a further three infants because of indeterminate sex (figure 1). A total of 10197 infants (53\% male) were included in the final analysis with a median (IQR) gestational age of 26.0 (24.9-27.1) weeks and birth weight of $0.81(0.68-0.96) \mathrm{kg}$ (table 1$)$. They were ventilated for a median of 10 (3-26) days and the ones that survived to discharge from neonatal care $(81 \%)$ were discharged at a median (IQR) PMA of 39.6 (37.6-42.1) weeks (table 1).

A four-cluster solution was found to be optimal (table 1). The clinical profiles for the four clusters are presented graphically in figures 2 and 3 . The boxplots of 


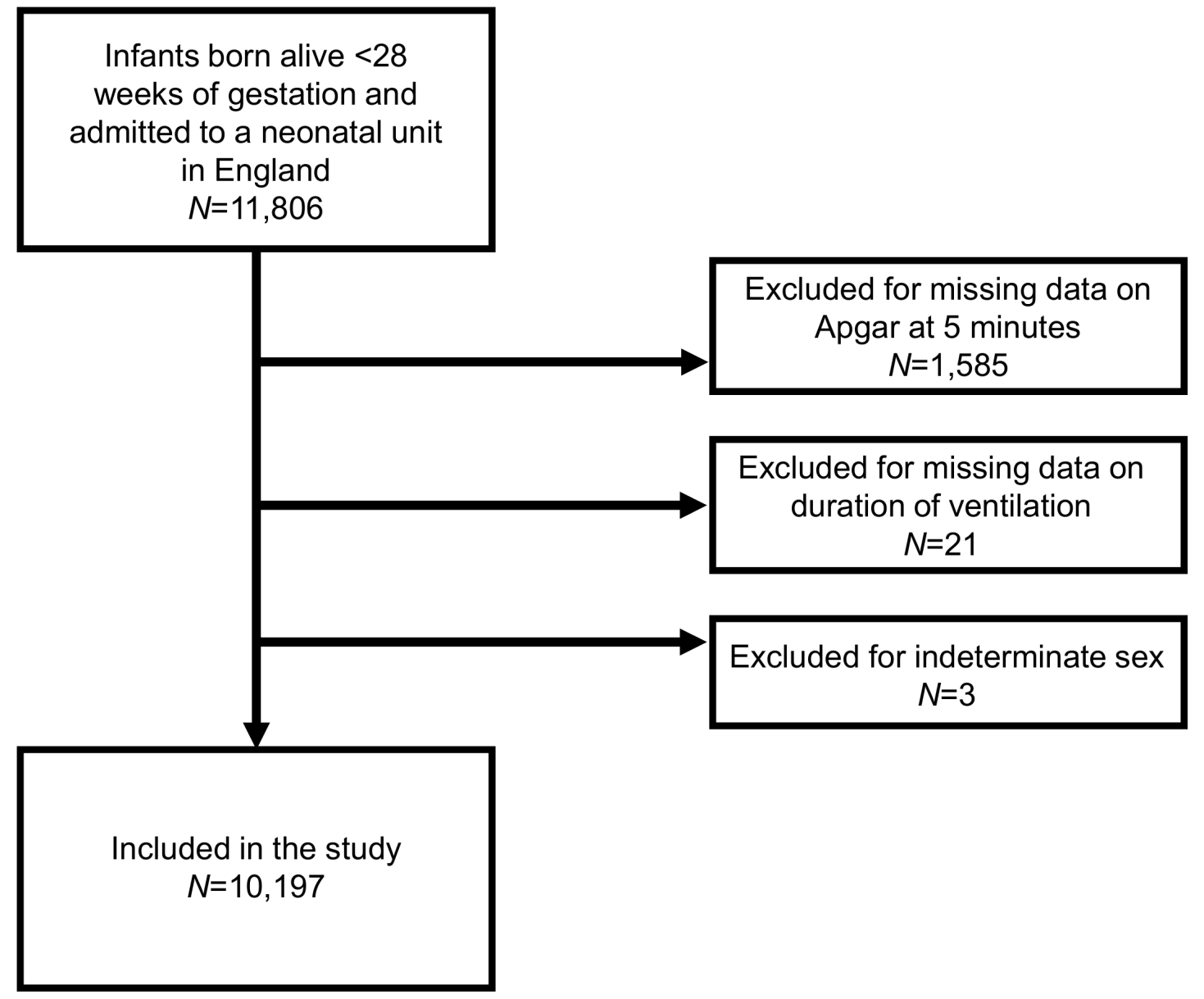

Figure 1 Flow diagram of the included infants.

the input parameters in the four clusters along with the birth weight a-score, the duration of PN and the PMA age at discharge are presented in figure 4.

Cluster 1 (intermediate) contained $27 \%$ of the population. The infants of cluster 1 had a lower median (IQR) gestational age (24.8 (24.1-25.2) weeks) compared with clusters 2 and 3 (27.0 (26.6-27.6) weeks and 25.4 (24.426.4) weeks respectively, $\mathrm{p}<0.001$ for both). The median Apgar score for infants of cluster 1 was higher compared with the infants of clusters 3 and 4 ( $p<0.001$ for both), but not of cluster 2 infants. Infants in cluster 1 were ventilated for a median (IQR) period of 21 (10-33) days, significantly longer that infants of clusters 2 and 3 (4 (2-10) and 9 (3-21) days, respectively, $\mathrm{p}<0.001$ for both) but significantly shorter than the infants of cluster 4 (60 (52-73) days, $\mathrm{p}<0.001)$. Infants of cluster 1 developed more often BPD (59\%) compared with infants of clusters 2 and 3 ( $48 \%$ and $42 \%$, respectively, $\mathrm{p}<0.001$ ) but less often than infants of cluster $4(89 \%, p<0.001)$. Infants of cluster 1 had lower mortality $(27 \%)$ compared with cluster $3(40 \%$, $\mathrm{p}<0.001)$ but higher mortality compared with cluster 2 $(8 \%, \mathrm{p}<0.001)$.

Cluster 2 (favourable) contained $47 \%$ of the population and had the highest median (IQR) gestational age (27.0 (26.6-27.6) weeks) compared with groups 1, 3 (25.4 (24.4-26.4) weeks, $\mathrm{p}<0.001)$ and 4 (24.4 (23.9-25.3) weeks, $\mathrm{p}<0.001)$. The median Apgar score for infants of cluster 2 was higher compared with the infants of clusters 3 and 4 ( $p<0.001$ for both). Infants in cluster $2 \mathrm{had}$ the shorter duration of ventilation with a median (IQR) period of 4 (2-10) days, compared with infants of cluster 1 (21 (10-33) days) ], cluster 3 (9 (3-21) days) and cluster 4 (60 (52-73) days, $\mathrm{p}<0.001$ for both). Infants of cluster 2 developed less often BPD (48\%) compared with infants of clusters 1 and 4 (59\% and $89 \%$, respectively, $\mathrm{p}<0.001$ for both), but more often than infants of cluster $3(42 \%$, $\mathrm{p}<0.001)$. Infants of cluster 2 had the lowest mortality $(8 \%)$ compared with all other clusters $(\mathrm{p}<0.001)$.

Cluster 3 (low Apgar) contained 17\% of the population and the infants with the lowest median (IQR) Apgar score (4 (2-5)) compared with all other groups ( $<<0.001$ for all). Their median (IQR) gestation (25.4 (24.4-26.4) weeks) was higher than clusters 1 and 4, but lower than cluster $2(\mathrm{p}<0.001$ for all). Infants of cluster $3 \mathrm{had}$ a shorter median (IQR) duration of ventilation (9 (3-21)) days, compared with infants of clusters 1 and 4 ( $<<0.001$ for both), but longer than infants of cluster 2 $(p<0.001)$. Infants of cluster 3 had the lower incidence of BPD $(42 \%)$ compared with infants of clusters 1, 2 and 4 (59\%, $48 \%$ and $89 \%$, respectively, $\mathrm{p}<0.001$ for all). Infants of cluster 3 had the highest mortality (40\%) compared with all other clusters $(\mathrm{p}<0.001)$. 


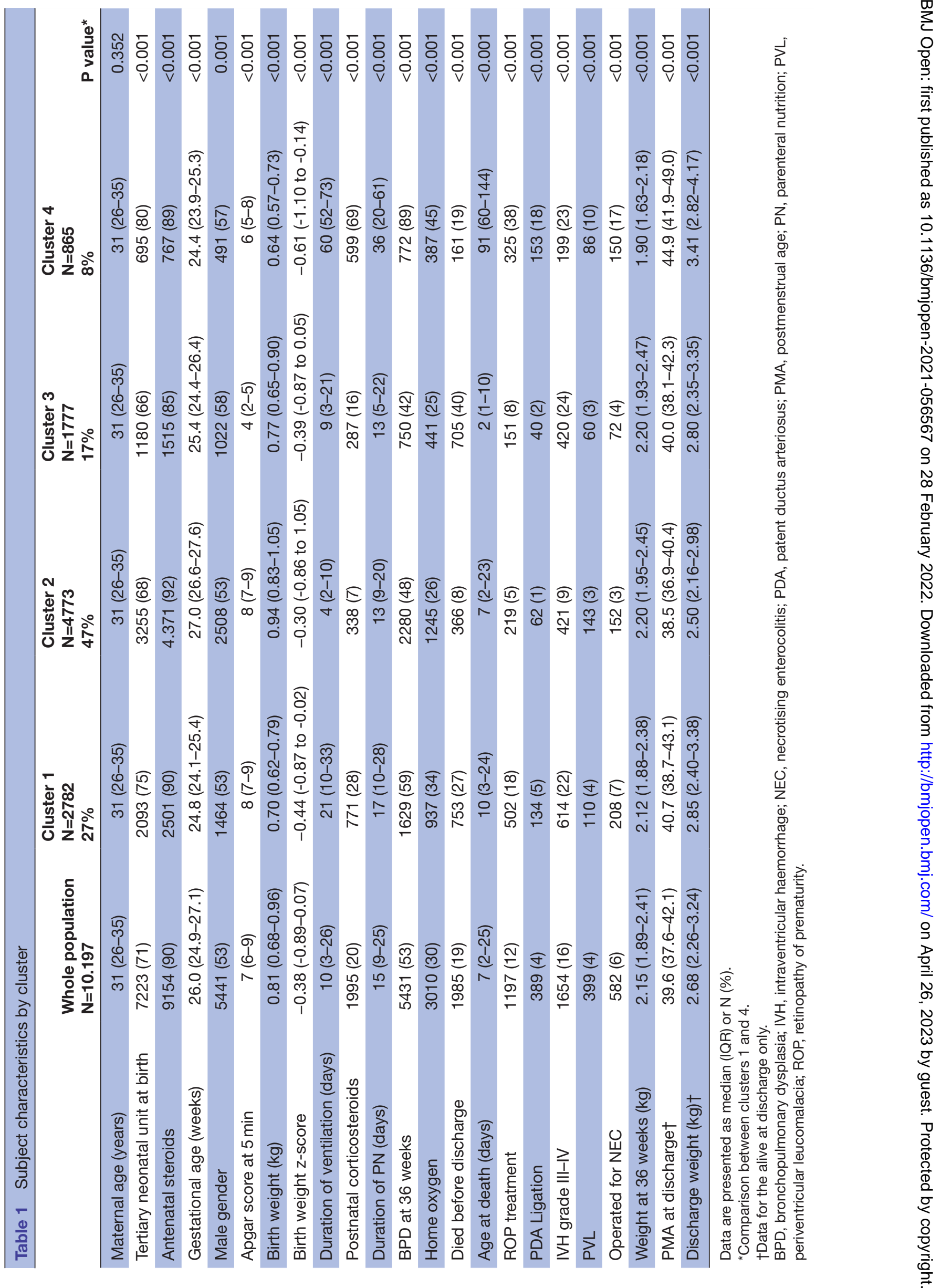



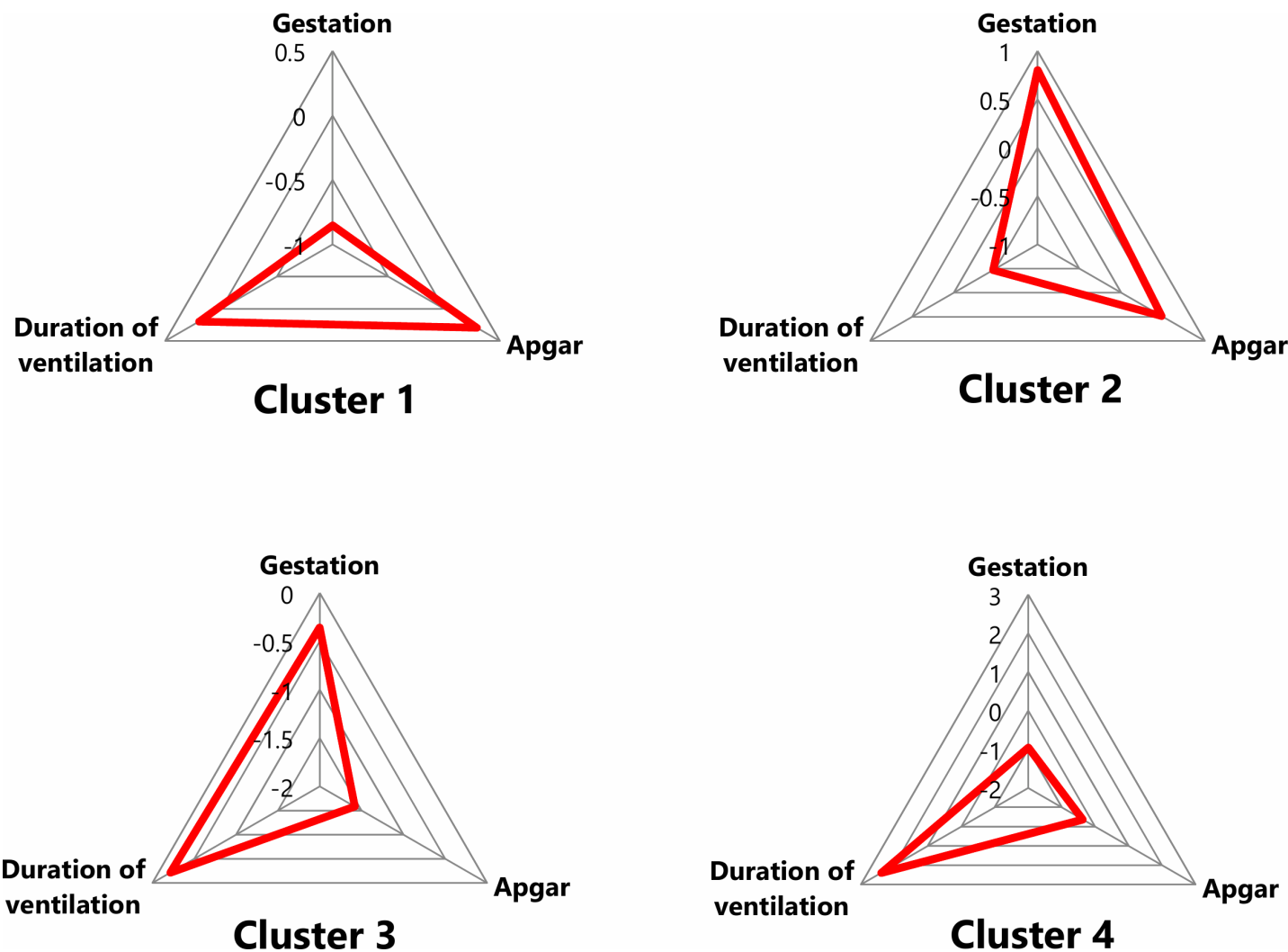

Figure 2 Radial plots showing the clinical profiles for the four clusters. Data are standardised (expressed as z-scores referenced to the whole cohort) and the spike points represent medians.

Cluster 4 (prolonged ventilation) had the smallest number of subjects $(8 \%)$, the lowest gestational age (24.4 (23.9-25.3) weeks) and the longest median (IQR) duration of ventilation (60 (52-73) days) compared with all other clusters $(\mathrm{p}<0.001$ for all). The median Apgar score for infants of cluster 4 was 6 (5-8), which was higher compared with the infants of cluster $3(\mathrm{p}<0.001)$ but lower than clusters 1 and 2 infants $(p<0.001$ for both).

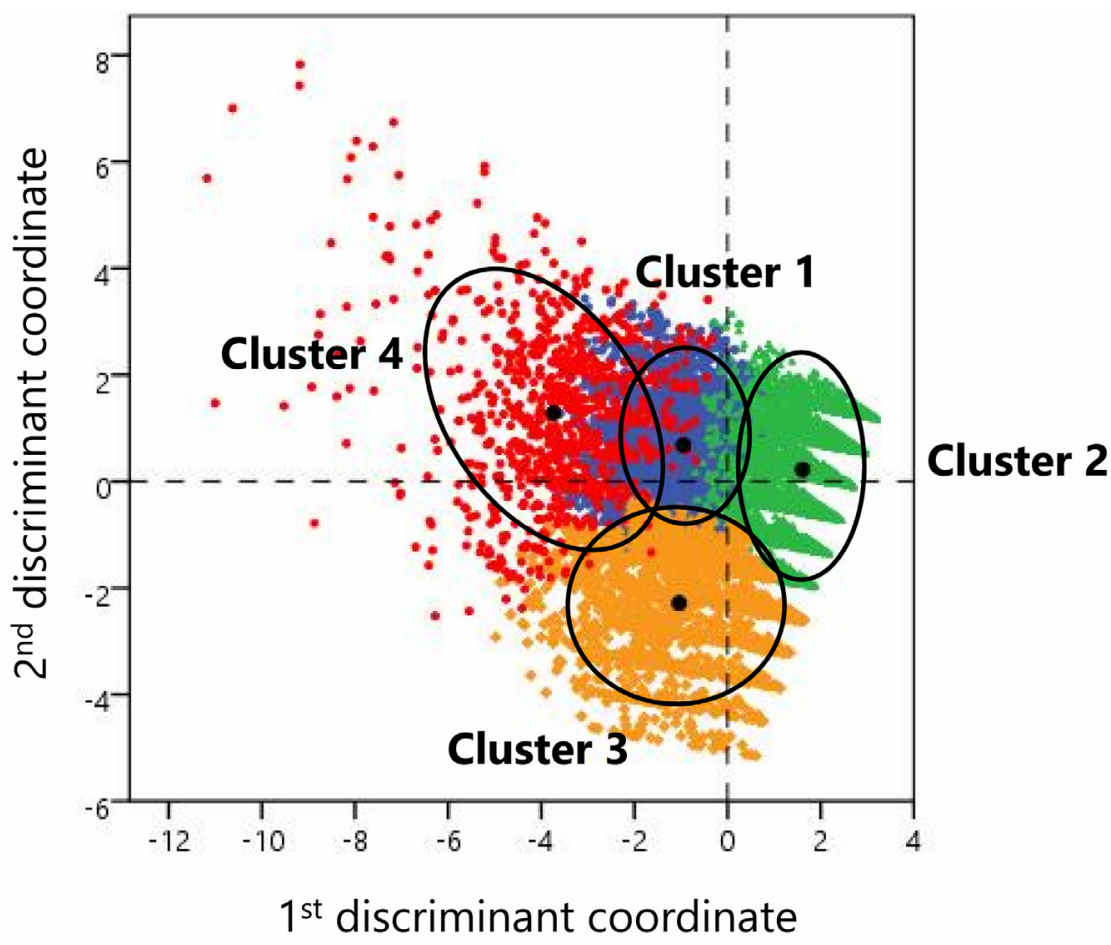

Figure 3 Discriminant two-dimensional plot of the clustering solution. The cluster centroids are presented as black circles. 

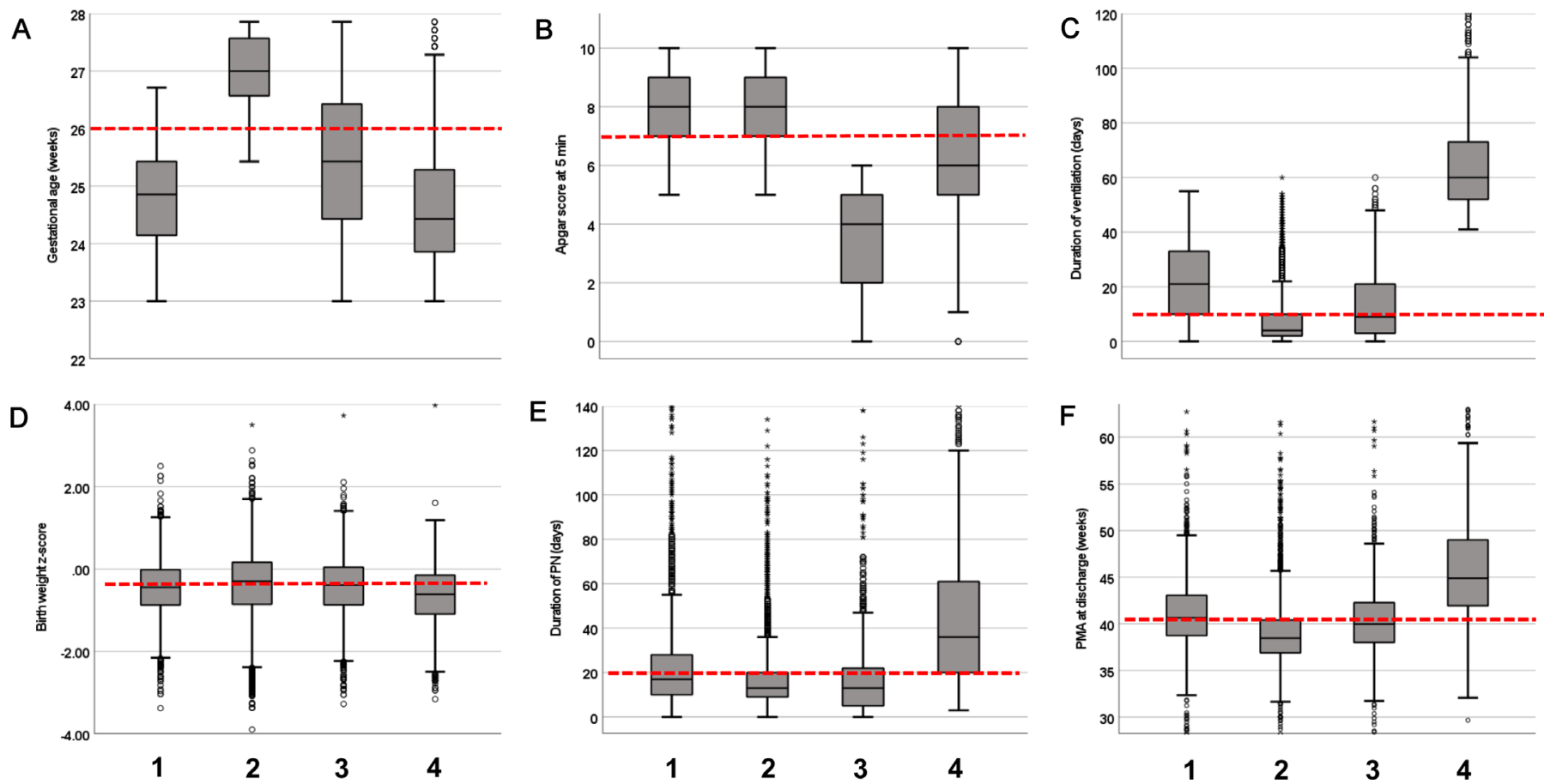

Figure 4 Boxplots of the gestational age (A), Apgar score at $5 \mathrm{~min}(\mathrm{~B})$, duration of ventilation (C), birth weight z-score $(D)$, duration of parenteral nutrition $(P N)(E)$ and postmenstrual age $(P M A)$ at discharge $(F)$ in the four clusters. The median value for each parameter for the whole population is depicted as a dashed line. The whiskers represent the maximum and minimum values. The asterisks are extremes (at least three box lengths from the median) and the circles are outliers (1.5 box lengths from the median).

Infants of cluster 4 had the highest rate of BPD (89\%) compared with infants of all other clusters $(\mathrm{p}<0.001$ for all). The mortality of the infants of cluster $4(19 \%)$ was lower than clusters 1 and 3 ( $\mathrm{p}<0.001$ for both) and higher than cluster $2(\mathrm{p}<0.001)$.

\section{DISCUSSION}

Using unsupervised learning to detect and describe distinct phenotypical groups of extremely preterm infants, we demonstrated that survival to discharge from neonatal care and other important neonatal outcomes differed significantly across these groups. We used readily available parameters to classify the extremely preterm infants into the four clusters.

Cluster 1 (intermediate) comprised infants with a combination of a low gestational age and a relatively long duration of invasive ventilation whose resuscitation was overall straightforward as demonstrated by a median Apgar of 8 at $5 \mathrm{~min}$ after birth. Nevertheless, they had a relatively high mortality and incidence of BPD. Cluster 2 (favourable) was the largest group and exhibited the best outcomes. It contained infants with the highest gestational age and birth weight z-score and the briefest duration of ventilation. Not surprisingly, they also had the lowest mortality and a relatively modest incidence of complications such as BPD. They were also the earliest to be discharged from neonatal care. The major clustering characteristic of the infants in cluster 3 was the low Apgar score. The poor condition at birth might explain why they had the highest mortality and rate of IVH compared with all other groups, but their relatively larger gestation and brief duration of ventilation might explain why they had the lowest incidence of BPD. Cluster 4 (prolonged ventilation) consisted of the most premature infants, with the longest duration of mechanical ventilation and highest rates of BPD. These infants were also discharged home at a later PMA than all other cluster infants.

Our results highlight two distinct within-cluster associations. First, poor condition at birth with subsequent high rates of mortality and IVH (cluster 3) and, second, the association of prolonged ventilation with BPD (cluster $4)$. Both these associations have been previously individually described in population studies: Jensen et al studied 3343 extremely low-birth weight infants and reported that among the survivors, exposure to a greater number of mechanical ventilation episodes was associated with a progressive increase in the risk of $\mathrm{BPD} .{ }^{23}$ Interestingly, the highest mortality in our study was not in the infants of the lower gestation at birth, but rather in the ones with the lowest Apgar score. The association of poor condition at birth with IVH and increased mortality is primarily attributed to the intrinsic fragility of the germinal matrix vasculature and the disturbance in the cerebral blood flow. ${ }^{24}$ It is also interesting to note that the infants of cluster 3, other than the highest mortality and lowest Apgar score, also had the lowest rate of antenatal steroids and the lowest incidence of birth in a tertiary centre. This is in agreement with previous studies that have 
highlighted that delivery outside a tertiary centre is associated with higher mortality and a lower uptake of antenatal steroids. ${ }^{26}$ Our study complements the literature by presenting these associations within distinct groups of the same population rather than via a unilateral relationship that does not take into account the other outcomes.

The choice of our input variables (gestational age, Apgar score at $5 \mathrm{~min}$ and duration of mechanical ventilation) was made on clinical grounds and has evidently major implications for our conclusions. We selected to use these three parameters because they are continuous and reliably available at a population level, while they also represent relatively distinct pathophysiological processes that are associated with impaired later outcomes. It is well described, for example, that the Apgar score is influenced by gestation and cannot be generally considered as evidence of asphyxia nor can it be used at an individual level to predict mortality or neurological outcomes. ${ }^{27}$ Alternative continuous variables to assess the condition at birth such as the cord pH or lactate could not be used, however, as these indices were not consistently inserted in the database and had a very high percentage of missing values. Similarly, the duration of ventilation was selected as a proxy for the severity of lung disease ${ }^{28}$ which also can be assessed relatively early chronologically as the median duration of ventilation in our population was 10 days.

To our knowledge, non-supervised learning and cluster analysis have not been previously applied exclusively to extremely preterm infants. Souza et al used K-means cluster analysis to explore clinical conditions associated with preterm birth and reported that 4150 preterm births were clustered in three groups of women, yet although some maternal characteristics differed among the clusters, maternal and neonatal outcomes did not. ${ }^{29}$ This might be explained by population differences, as this was predominantly a maternity study in which the neonatal outcomes were not considered as input variables in the unsupervised models. Greenbury et al performed clustering on daily nutritional intakes in premature infants of less than 32 weeks of gestation and reported different nutritional clusters, which were heterogeneous in size, with some showing common interpretable clinical practices. They also identified a relationship between nutritional practice and outcomes such as BPD, with provision of human milk being a protective factor against developing BPD. ${ }^{30}$

Our classification could be clinically useful in further understanding the pathophysiology of extremely preterm morbidity and mortality and possibly in targeting selected subgroups of infants for specific interventions. For example, for cluster 4 , such interventions might include administration of postnatal corticosteroids to assist earlier weaning from invasive respiratory support, ${ }^{31}$ more gentle resuscitation methods, avoidance of mechanical ventilation by newer non-invasive techniques ${ }^{32}$ and use of less invasive surfactant administration, which has been shown to reduce the incidence of BPD. ${ }^{33}$ The within-group association of increased mortality and birth outside a tertiary hospital in cluster 3 could also strengthen the argument for the centralisation of the care of extreme preterms concentrating on high-volume perinatal centres. ${ }^{34}$

Our study has strengths and some limitations. To our knowledge, this is the first study to use unsupervised learning to classify extremely preterm infants in different phenotypical groups. A further strength of our study was that we used the whole population rather than a representative sample and therefore, by avoiding inclusion bias, our results are more generalisable. We should clarify that our analysis is not an early predictive tool that could be applied at birth, as we have included the duration of ventilation as an input parameter, which comes later in the life of an extremely preterm infant compared with the Apgar score or the gestational age. As such, our study is aiming to describe the interaction of different pathophysiological phenomena at a population level rather than provide individualised prediction of outcomes at birth. We should also note that our study only included infants from a recent 5-year period rather than a longer period that would extend more in the past. Our tighter time period, though, would better correspond to current standards of neonatal care. We did not include in our analysis information on whether preterm birth was spontaneous or on the duration of rupture of membranes as the amount of missing data in these categories was very high at a national level, even more so in non-tertiary units.

In conclusion, we have used readily available clinical parameters to classify extremely preterm infants into distinct phenotypic groups. The ensuing groups had differing neonatal outcomes such as survival to discharge from neonatal care and BPD.

Contributors TD: conceived the study, participated in data analysis, wrote the first version of the manuscript and is the guarantor of the manuscript, EEW: acquired and analysed the dataset and critically reviewed the manuscript. $\mathrm{CH}$ : participated in data analysis and critically revised the manuscript; AG: participated in study design, supervised the project and critically revised the manuscript.

Funding Emma Williams was supported by a grant from the Charles Wolfson Charitable Trust and a non-conditional educational grant from SLE. This research was supported by the National Institute for Health Research (NIHR) Biomedical Research Centre at Guy's and St Thomas' NHS Foundation Trust and King's College London. The views expressed are those of the authors and not necessarily those of the NHS, the NIHR or the Department of Health.

Competing interests Professor Greenough has held grants from various manufacturers (Abbot Laboratories, Medlmmune) and ventilator manufacturers (SLE). Professor Greenough has received honoraria for giving lectures and advising various manufacturers (Abbot Laboratories, Medlmmune) and ventilator manufacturers (SLE). Professor Greenough is currently receiving a non-conditional educational grant from SLE.

Patient and public involvement Patients and/or the public were not involved in the design, or conduct, or reporting, or dissemination plans of this research.

\section{Patient consent for publication Not applicable.}

Ethics approval A predefined dataset was acquired from the National Neonatal Research Database (NNRD), Imperial College London, UK. The NNRD is approved by the National Research Ethics Service (10/H0803/151), Confidentiality Advisory Group of the Health Research Authority (8-05[f]/2010) and the Caldicott Guardians and Lead Clinicians of contributing hospitals. As the study used data held in an existing database, participation did not require approval from individual Trusts, but only from the NHS Trust holding the database (Chelsea and Westminster NHS Foundation Trust) which was obtained. The primary study was approved by the West Midlands-Edgbaston Research Ethics Committee (REC reference: 19/WM/0172) 
and the UK Health Research Authority (HRA) (IRAS project ID: 259225). The research was conducted ethically in accordance with the World Medical Association Declaration of Helsinki.

Provenance and peer review Not commissioned; externally peer reviewed.

Data availability statement Data are available upon reasonable request.

Open access This is an open access article distributed in accordance with the Creative Commons Attribution Non Commercial (CC BY-NC 4.0) license, which permits others to distribute, remix, adapt, build upon this work non-commercially, and license their derivative works on different terms, provided the original work is properly cited, appropriate credit is given, any changes made indicated, and the use is non-commercial. See: http://creativecommons.org/licenses/by-nc/4.0/.

ORCID iDs

Theodore Dassios http://orcid.org/0000-0001-5258-5301

Anne Greenough http://orcid.org/0000-0002-8672-5349

\section{REFERENCES}

1 Chawanpaiboon S, Vogel JP, Moller A-B, et al. Global, regional, and national estimates of levels of preterm birth in 2014: a systematic review and modelling analysis. Lancet Glob Health 2019;7:e37-46.

2 Stahlmann N, Eisemann N, Thyen U, et al. Long-term health outcomes and health-related quality of life in adolescents from a cohort of extremely premature infants born at less than 27 weeks of gestation in northern Germany. Neuropediatrics 2016;47:388-98.

3 Glass HC, Costarino AT, Stayer SA. Outcomes for extremely premature infants. Anes Analg 2015;120:1337-51.

4 Beam AL, Fried I, Palmer N, et al. Estimates of healthcare spending for preterm and low-birthweight infants in a commercially insured population: 2008-2016. J Perinatol 2020;40:1091-9.

5 Lakshmanan A, Agni M, Lieu T, et al. The impact of preterm birth $<37$ weeks on parents and families: a cross-sectional study in the 2 years after discharge from the neonatal intensive care unit. Health Qual Life Outcomes 2017;15:38.

6 Cheong JL, Spittle AJ, Burnett AC, et al. Have outcomes following extremely preterm birth improved over time? Semin Fetal Neonatal Med 2020;25:101114.

7 Patel RM. Short- and long-term outcomes for extremely preterm infants. Am J Perinatol 2016;33:318-28.

8 Dassios T, Williams EE, Hickey A, et al. Bronchopulmonary dysplasia and postnatal growth following extremely preterm birth. Arch Dis Child Fetal Neonatal Ed 2021;106:386-391. [Epub ahead of print].

9 Jensen EA, Schmidt B. Epidemiology of bronchopulmonary dysplasia. birth defects research Part a. Clin Molecular Teratol 2014;100:145-57.

10 Alonso-Betanzos A, Bolon-Canedo V. Big-data analysis, cluster analysis, and machine-learning approaches. Adv Exper Med Biol 2018;1065:607-26.

11 Lunt A, Mortimer L, Rees D, et al. Heterogeneity of respiratory disease in children and young adults with sickle cell disease. Thorax 2018;73:575-7.

12 MacBean V, Lunt A, Drysdale SB, et al. Predicting healthcare outcomes in prematurely born infants using cluster analysis. Pediatr Pulmonol 2018;53:1067-72.

13 Jobe AH, Bancalari E. Bronchopulmonary dysplasia. Am J Respir Crit Care Med 2001;163:1723-9.

14 Higgins RD, Jobe AH, Koso-Thomas M, et al. Bronchopulmonary dysplasia: executive summary of a workshop. J Pediatr 2018;197:300-8.

15 Patel RM, Ferguson J, McElroy SJ, et al. Defining necrotizing enterocolitis: current difficulties and future opportunities. Pediatr Res 2020;88:10-15.
16 Heuchan AM, Clyman RI. Managing the patent ductus arteriosus: current treatment options. Arch Dis Child Fetal Neonatal Ed 2014;99:F431-6.

17 Papile LA, Burstein J, Burstein R, et al. Incidence and evolution of subependymal and intraventricular hemorrhage: a study of infants with birth weights less than 1,500 GM. J Pediatr 1978;92:529-34.

18 Gotardo JW, Volkmer NdeFV, Stangler GP, et al. Impact of periintraventricular haemorrhage and periventricular leukomalacia in the neurodevelopment of preterms: a systematic review and metaanalysis. PLoS One 2019;14:e0223427.

19 Wright CM, Williams AF, Elliman D, et al. Using the new UK-WHO growth charts. BMJ 2010;340:c1140.

20 Garcia-Aymerich J, Gómez FP, Benet M, et al. Identification and prospective validation of clinically relevant chronic obstructive pulmonary disease (COPD) subtypes. Thorax 2011;66:430-7.

21 Everitt BS, Landau S, Leese M. Cluster analysis. 4th edn. London: Arnold, 2001.

22 Milligan GW, Cooper MC. An examination of procedures for determining the number of clusters in a data set. Psychometrika 1985;50:159-79.

23 Jensen EA, DeMauro SB, Kornhauser M, et al. Effects of multiple ventilation courses and duration of mechanical ventilation on respiratory outcomes in extremely low-birth-weight infants. JAMA Pediatr 2015;169:1011-7.

24 Ballabh P. Intraventricular hemorrhage in premature infants: mechanism of disease. Pediatr Res 2010;67:1-8.

25 Szpecht D, Szymankiewicz M, Nowak I, et al. Intraventricular hemorrhage in neonates born before 32 weeks of gestationretrospective analysis of risk factors. Childs Nerv Syst 2016;32:1399-404.

26 Helenius K, Longford N, Lehtonen L. Neonatal data analysis unit and the United Kingdom neonatal collaborative. Association of early postnatal transfer and birth outside a tertiary hospital with mortality and severe brain injury in extremely preterm infants: observational cohort study with propensity score matching. BMJ 2019;367:15678.

27 , American Academy of Pediatrics Committee on Fetus and Newborn, American College of Obstetricians and Gynecologists Committee on Obstetric PracticeWatterberg KL, Aucott S, Benitz WE. The Apgar score. Pediatrics 2015;136:819-22.

28 Dassios T, Williams EE, Hickey A, et al. Duration of mechanical ventilation and prediction of bronchopulmonary dysplasia and home oxygen in extremely preterm infants. Acta Paediatr 2021;110:2052-8.

29 Souza RT, Cecatti JG, Passini R, et al. Cluster analysis identifying clinical phenotypes of preterm birth and related maternal and neonatal outcomes from the Brazilian multicentre study on preterm birth. Int J Gynaecol Obstet 2019;146:110-7.

30 Greenbury SF, Ougham K, Wu J, et al. Identification of variation in nutritional practice in neonatal units in England and association with clinical outcomes using agnostic machine learning. Sci Rep 2021;11:7178.

31 Doyle LW, Cheong JL, Ehrenkranz RA, et al. Late (> 7 days) systemic postnatal corticosteroids for prevention of bronchopulmonary dysplasia in preterm infants. Cochrane Database Syst Rev 2017; 10:CD001145.

32 Sand L, Szatkowski L, Kwok T'ng Chang, et al. Observational cohort study of changing trends in non-invasive ventilation in very preterm infants and associations with clinical outcomes. Arch Dis Child Fetal Neonatal Ed 2022;107:150-5.

33 Aldana-Aguirre JC, Pinto M, Featherstone RM, et al. Less invasive surfactant administration versus intubation for surfactant delivery in preterm infants with respiratory distress syndrome: a systematic review and meta-analysis. Arch Dis Child Fetal Neonatal Ed 2017;102:F17-23.

34 Walther F, Küster DB, Bieber A, et al. Impact of regionalisation and case-volume on neonatal and perinatal mortality: an umbrella review. BMJ Open 2020;10:e037135. 